American Journal of Agricultural and Biological Science 2(1): 31-35, 2007

ISSN 1557-4989

(C) 2007 Science Publications

\title{
Intranasal Administration of Quercetin Liposome Decreases Anxiety-like Behavior and Increases Spatial Memory
}

\author{
${ }^{1}$ Jintanaporn Wattanathorn, ${ }^{2}$ Wathita Phachonpai, ${ }^{3}$ Aroonsri Priprem and ${ }^{4}$ Saengrawee Suthiparinyanont \\ ${ }^{1}$ Department of Physiology, Faculty of Medicine, Khon Kaen University \\ Thailand, 40002, 66-43-348394 \\ ${ }^{2}$ Department of Physiology (Neuroscience Program) and Graduate School, Faculty of Medicine and \\ Graduate School, Khon Kaen University, Thailand, 40002 \\ ${ }^{3}$ Department of Technology and Pharmaceutical Sciences, Faculty of Pharmaceutical Sciences, \\ Khon Kaen University, Thailand, 40002 \\ ${ }^{4}$ Department of Technology and Pharmaceutical Sciences and Graduate School, Khon Kaen University, \\ Thailand, 40002
}

\begin{abstract}
At present, the effects of quercetin liposome on anxiolytic activity and cognitive function are unclearly known. In this study, male Wistar rats were administered quercetin liposome via intranasal route once daily continually for 4 weeks. The anxiolytic activity and cognitive enhancing effect were determined after single administration, 1, 2, 3 and 4 weeks of treatment using elevated plus maze test and Morris water maze test respectively. The results showed that quercetin liposome significantly increased both number of opened arm entry and time spent in the opened arm. In addition, the quercetin liposome also decreased escape latency and increased retention time markedly. Therefore, our results demonstrated the anxiolytic activity and cognitive enhancing effect of quercetin liposome and suggested that it might be used as a novel therapeutic strategy for anxiety disorder and cognitive deficit condition.
\end{abstract}

Key words: Quercetin; liposome; anxiolytic activity; cognitive function; intranasal route; elevated plus maze; morris water maze test

\section{INTRODUCTION}

Quercetin is one of the most abundant flavonoids present in fruits and vegetables ${ }^{[1]}$ which possess variety of pharmacological activities including the abilities to prevent against many disorders such as cancer, cardiovascular and neurodegeneration diseases ${ }^{[2]}$. In addition, oral administration of quercetin was also able to improve learning and memory ability ${ }^{[3]}$.

Previous studies demonstrated that the efficacy of the substance seemed to depend on many factors including the route of administration. It is important to realize that after oral administration, this substance is poorly absorbed from the intestine, highly metabolized, or rapidly eliminated ${ }^{[4]}$. Therefore, new therapeutic approach such as liposome encapsulation of quercetin is warrant. In addition, it was also reported that nasal administration offered many benefits including bypass the hepatic presystemic metabolism, simple dose adjustments, constant absorption and highly convenient for the patient administration ${ }^{[5]}$. Moreover, nasal delivery was proposed to be the alternative administration route to target drugs directly to brain via the olfactory neurons ${ }^{[6,7]}$, therefore, this provides more opportunity for quercetin to enter the central nervous system and then act on central nervous system (CNS) to promote learning and memory with highly efficiency. Based on the information mentioned above, the effects of quercetin liposome on anxiolytic activity and cognitive function are investigated in this study.

\section{MATERIALS AND METHODS}

Animals: Male Wistar rats at 16 weeks old weight approximate 180-200 gms (National Animal Center, Salaya, Nakorn Pathom) were housed in group of 5 per cage in standard metal cages at $22 \pm 2^{\circ} \mathrm{C}$ on $10: 14 \mathrm{~h}$ light - dark cycle with food and water available at all

Corresponding Author: $\quad$ Jintanaporn Wattanathorn, Department of Physiology, Faculty of Medicine, Khon Kaen University, Thailand, 40002 
time. The animals were kept in the animal care facility of Faculty of Medicine, Khon Kaen University in the compliment of institutional and European Community (EEC directive of 1986; 86/609/EEC) guideline.

Preparation of liposome: High-purity egg L- $\alpha-$ phosphatidylcholine, Type XVI-E (EPC), cholesterol (CHOL), and quercetin dehydrate (98\% HPLC purity) were purchased from Sigma (Barcelona, Spain) and prepared as quercetin encapsulated liposome. Freshly prepared quercetin liposome, containing $0.5 \mathrm{mg}$ of quercetin in $20 \mu \mathrm{l}$ (dose $.06 \mathrm{mg} / \mathrm{kg} \mathrm{BW}$ ) being drawn into a micropipette, were administered to the right nasal cavity of each rat which was then holding still for 20 seconds to ensure the flow and contact of the quercetin liposome to the nasal cavity whereas the control group received liposome without quercetin at the same volume once daily for 4 weeks. The rats were treated at lease 20 minutes prior to the daily experiment.

Elevated plus maze test: The anxiolytic effect was assessed using the elevated plus maze which consisted of open arms $(50 \times 10 \mathrm{~cm})$ and two enclosed arms $(50 \times$ $10 \mathrm{~cm}$ ) with $40 \mathrm{~cm}$ high walls, extending from a central platform $(10 \times 10 \mathrm{~cm})$. The arms were connected with a central square, $10 \times 10 \mathrm{~cm}$, to give the apparatus a plus sign appearance. The maze was raised to a height of $50 \mathrm{~cm}$ above floor. The maze floor and walls were constructed from dark opaque wood. Each rat was placed on the center of the platform facing an enclosed arm. Animals were tested individually and only once for 5 minutes. The number of opened arm entries and time spent in the opened arms were recorded as anxiolytic indices.

Morris water maze test: The rats were also determined the spatial memory using Morris water maze test maze consisted of a metal pool $(170 \mathrm{~cm}$ in diameter $\times 58 \mathrm{~cm}$ tall $)$ filled with tap water $\left(25^{\circ} \mathrm{C}, 40\right.$ $\mathrm{cm}$ deep) divided into 4 quadrants. In the center of 1 quadrant was a removable escape platform below the water level and covered with a nontoxic milk powder. The pool was divided into 4 quadrants (NE, NW, SE, and SW) by two imaginary lines crossing the center of the pool. For each animal, the location of invisible platform was placed at the center of one quadrant and remained there throughout training. The rats must memorize the platform location in relation to various environmental cues because there was nothing directly showed the location of the escape platform in and outside the pool. Therefore, the placement of the water tank and platform were the same in all acquisition trials. Each rat was gently placed in the water facing the wall of the pool from one of the four starting points $(\mathrm{N}, \mathrm{E}, \mathrm{S}$, or $\mathrm{W}$ ) along the perimeter of the pool, and the animal was allowed to swim until it found and climbed onto the platform. During training session, the rat was gently placed on the platform by experimenter when it could not reach the platform in $60 \mathrm{~S}$. In either case, the subject was left on the platform for $15 \mathrm{~s}$ and removed from the pool. The time for animals to climb on the hidden platform was recorded as escape latency or acquisition time. On the next day, the rats were also exposed to the test again except that the platform was removed and the retention time or the time that the animal spent to swim around the previous location of platform before removing the platform was recorded.

Spontaneous motor behavior: All animals were assessed spontaneous motor behaviors including grooming and rearing behaviors for 5 minutes. The performance was assessed 60 minutes after the substances administration

Statistical analysis: The statistical analysis was performed using analysis of variance (ANOVA) followed by Duncan's test. The p-value less than 0.05 is considered as statistically significance.

\section{RESULTS}

The present results showed that liposome containing vehicle or polyethyleneglycol did not produce significant changes in all parameters assessed in this study compared to control group as shown in Fig. 1 to 7.

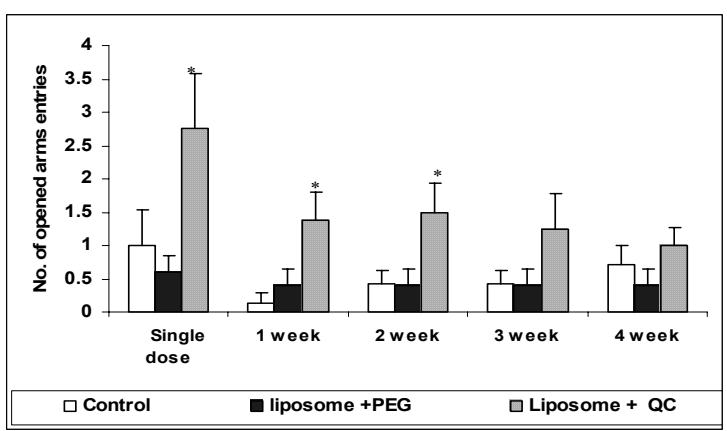

Fig. 1: Number of opened arm entries of rats in various groups including: control, liposome containing vehicle or polyethyleneglycol (liposome+PEG) and liposome containing quercetin (liposome $+\mathrm{QC}$ or QCL) via nasal route once daily for 4 weeks. The performance was assessed after single administration, 1, 2, 3 and 4 week after treatment. Data are presented as mean \pm S.E.M. ( $\mathrm{n}=8$ /group) ${ }^{*}$ p-value $<0.001$ compared with liposome+PEG. 


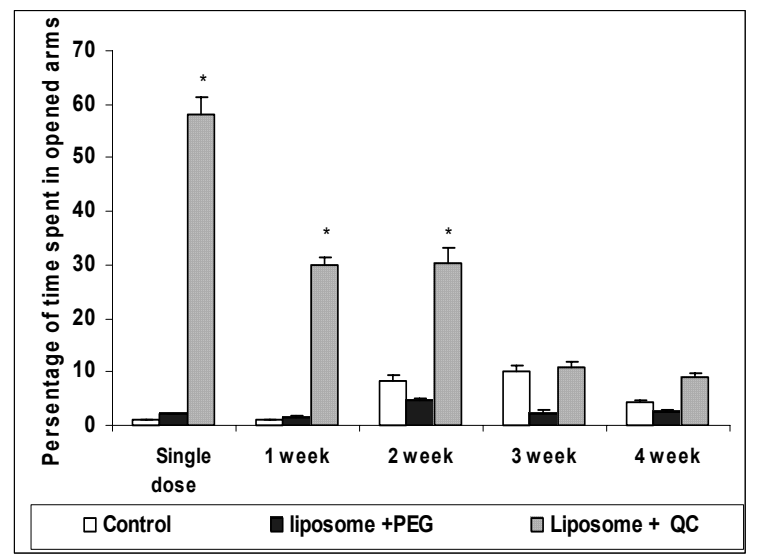

Fig. 2:Percentage of time spent in opened arm of rats in various groups including: control, liposome containing vehicle or polyethyleneglycol (liposome+PEG) and liposome containing quercetin (liposome+QC or QCL) via nasal route once daily for 4 weeks. The performance was assessed after single administration, 1, 2, 3 and 4 week after treatment. Data are presented as mean \pm S.E.M. (n=8/group) ${ }^{*}$ p-value $<0.001$ compared with liposome+PEG.

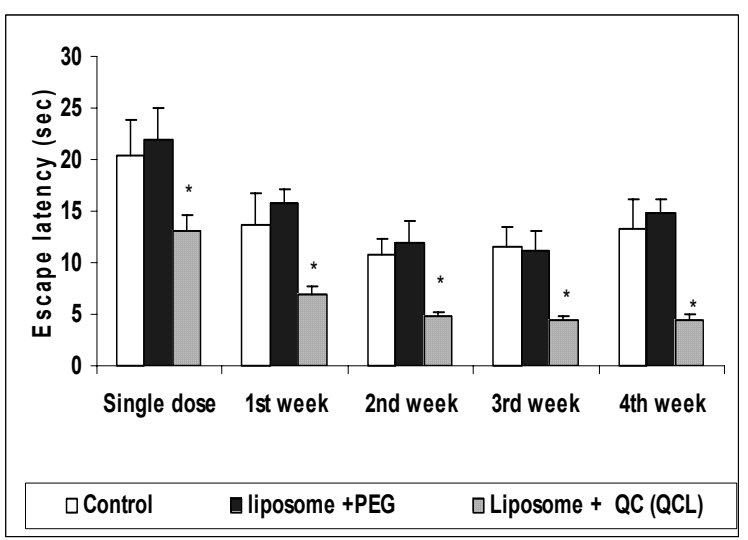

Fig. 3: Escape latency of rats in various groups including: control, liposome containing vehicle or polyethyleneglycol (liposome+PEG) and liposome containing quercetin (liposome $+\mathrm{QC}$ or $\mathrm{QCL}$ ) via nasal route once daily for 4 weeks. The performance was assessed after single administration, 1, 2, 3 and 4 week after treatment. Data are presented as mean \pm S.E.M. ( $\mathrm{n}=8 /$ group) ${ }^{*} \mathrm{p}$ value $<0.001$ compared with liposome+PEG.

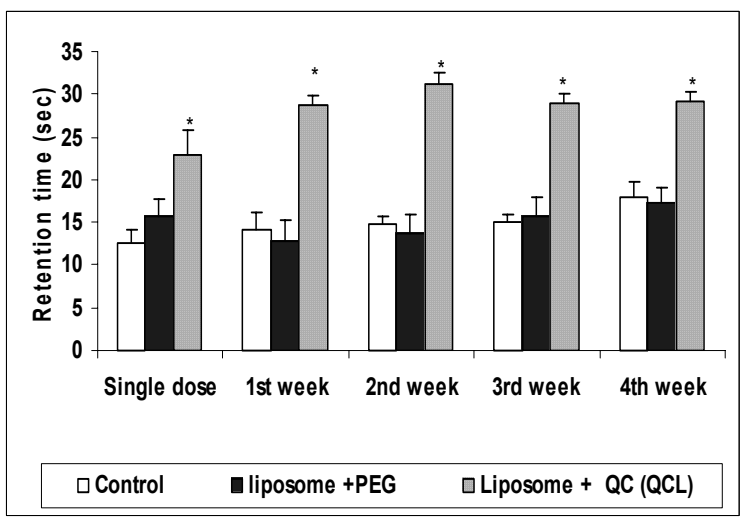

Fig. 4:Retention time of rats in various groups including: control, liposome containing vehicle or polyethyleneglycol (liposome+PEG) and liposome containing quercetin (liposome $+\mathrm{QC}$ or $\mathrm{QCL}$ ) via nasal route once daily for 4 weeks. The performance was assessed after single administration, 1, 2, 3 and 4 week after treatment. Data are presented as mean \pm S.E.M. (n=8/group) ${ }^{*}$ p-value $<0.001$ compared with liposome + PEG.

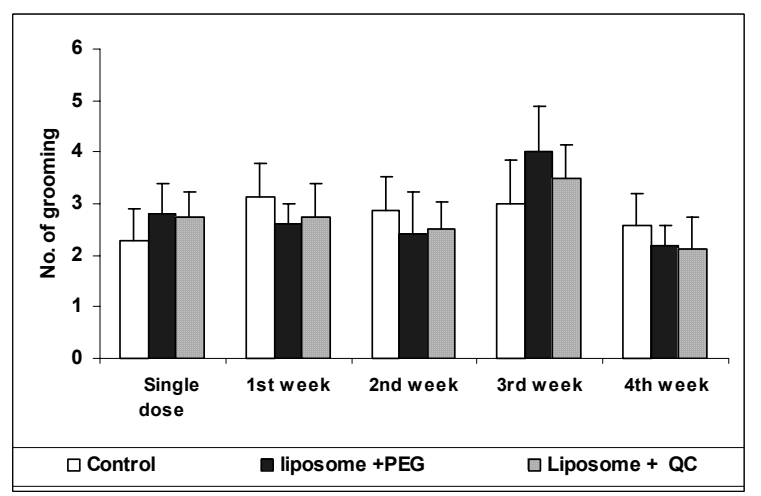

Fig. 5: Grooming behaviors of rats in various groups including: control, liposome containing vehicle or polyethyleneglycol (liposome+PEG) and liposome containing quercetin (liposome $+\mathrm{QC}$ or $\mathrm{QCL}$ ) via nasal route once daily for 4 weeks. The performance was assessed after single administration, 1, 2, 3 and 4 week after treatment. Data are presented as mean \pm S.E.M. ( $\mathrm{n}=8 /$ group)

The quercetin liposome treated group significantly increased both the number of opened arm entry and 


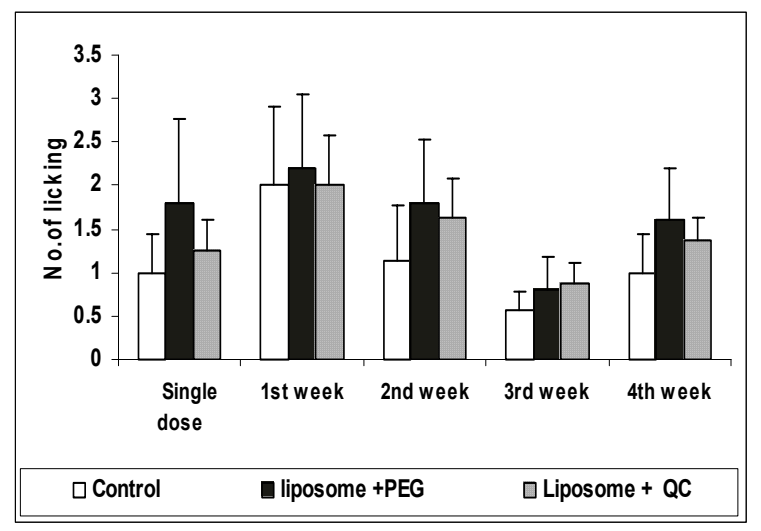

Fig. 6: Licking behaviors of rats in various groups including: control, liposome containing vehicle or polyethyleneglycol (liposome+PEG) and liposome containing quercetin (liposome $+\mathrm{QC}$ or $\mathrm{QCL}$ ) via nasal route once daily for 4 weeks. The performance was assessed after single administration, 1, 2, 3 and 4 week after treatment. Data are presented as mean \pm S.E.M. ( $\mathrm{n}=8$ /group)

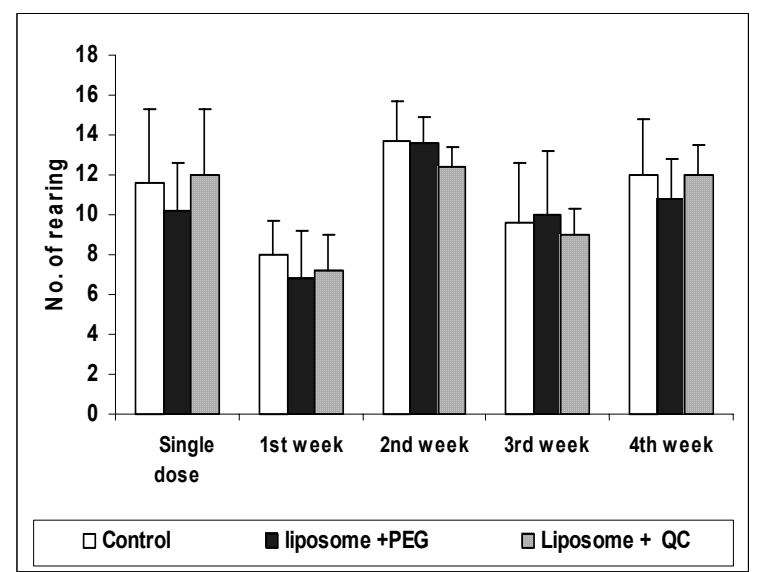

Fig. 7: Rearing behaviors of rats in various groups including: control, liposome containing vehicle or polyethyleneglycol (liposome+PEG) and liposome containing quercetin (liposome+QC or QCL) via nasal route once daily for 4 weeks. The performance was assessed after single administration, 1, 2, 3 and 4 week after treatment. Data are presented as mean \pm S.E.M. ( $\mathrm{n}=8 /$ group)

time spent in the opened arm after single dose of administration, 1 and 2 weeks of treatment. The increase treatment duration further to 3 and 4 weeks failed to show the significant changes of both parameters as shown in Fig. 1 and 2 (p-value $<.001$ all). Moreover, the quercetin liposome also exerted cognitive enhancing effect on spatial memory. The escape latencies were significantly decrease whereas the retention time were markedly increased as shown in Fig. 3 and 4 (p-value $<.001$ all).

Figure 5-7 showed that liposome containing quercetin failed to produce significant changes in spontaneous motor behaviors including grooming, licking and rearing behaviors.

Taken together, quercetin liposome exerted anxiolytic-like activity and cognitive enhancing effect without changes in spontaneous motor behaviors, therefore, the anxiolytic and cognitive enhancing effects induced by quercetin liposome were not false positive.

\section{DISCUSSION}

It has been previously reported that quercetin is a bioflavonoid that possess potent antioxidant and numerous effects in the central nervous system including antioxidant, neuroprotection, anti-anxiety and cognitive enhancing effect ${ }^{[6,8,9,]}$. However, quercetin is poorly absorbed and the distribution of conventional quercetin in the rat brain after orally administration is very low $^{[10]}$ due to the poorly absorbed, highly metabolized $^{[11]}$ and the penetration difficulty through blood brain barrier ${ }^{[12]}$.

Previous study had demonstrated that nasal administration offered the potential direct transfer of substances from the olfactory mucosa along the olfactory pathway to the central nervous system ${ }^{[6,7]}$ by bypassing the blood-brain barrier (BBB), which prevented some CNS-active drugs from reaching the brain $^{[13]}$. It was suggested that substances could be absorbed via the olfactory route by two different mechanisms. The very lipid soluble compounds could diffuse or retrograde transport along the axon to the olfactory bulbs and diffuse to the rest of the brain whereas the hydrophilic and semi-lipophilic substances could be transported into the cerebrospinal fluid via the subarachnoid space which extended along the olfactory nerves to the basolateral side of the olfactory epithelium. The first route of transport was relatively slow and was probably not relevant in terms of drug administration while the second route enabled relatively quick absorption to the cerebrospinal fluid of the substances.

Recently, it has been reported that liposome is an effective convection enhance delivery system to the brain because it can avoid both the fast elimination and difficulty in penetrating the blood brain barrier seen in systemic application ${ }^{[14]}$. 
Our results demonstrated that quercetin liposome provided several advantages including the effective at very much low dose and the significant improvement in both anxiolytic and cognitive enhancing effect were found very soon after single administration. The anxiolytic and cognitive enhancing effects had previously shown to be associated with the alteration of various neurotransmitters including gamma aminobutyric acid $(\mathrm{GABA})^{[6]}$ and serotonin $^{[15]}$ repectively. Therefore, based on the advantages of both liposome and nasal delivery, we do suggest that quercetin liposome may possibly be transported primarily via the cerebrospinal fluid (CSF) and exert its anxiolytic and cognitive enhancing effects via the alteration of gamma aminobutyric acid and serotonin ${ }^{[15]}$ respectively. However, further studies about the precise changes underlying these effects are still required.

The prolonged quercetin treatment duration failed to show significant anxiolytic effect. This might be related to the neuroadaptation of the neuronal pathway which played important role in anxiety.

In conclusion, nasal administration of quercetin encapsulated liposome is a potential novel therapeutic approach to improve anxiety-like behavior and cognitive function.

\section{ACKNOWLEDGEMENT}

The National Research Council of Thailand and Integrated Nanotechnology Research Center, Khon Kaen University, Thailand supported this study.

\section{REFERENCES}

1. Chu, Y., 2000. Flavonoid contents of several vegetables and their antioxidant activity. J. Sci. Food Agricul., 80: 561-566.

2. Hertog, M.G.L., P.C.H. Hollman and M.B. Katan, 1992. Content of potentially anticarcinogenic flavonoids of 28 vegetables and 9 fruits commonly consumed in the Netherlands. J Agric Food Chem., 40: 2379-2383.

3. Lu J., Y.L Zheng, L. Luo, D.M. Wu, D.X. Sun and Y.J. Feng, 2006. Quercetin reverses D-galactose induced neurotoxicity in mouse brain. Behav Brain Res. , 171: 251-60;

4. Manach C.,A. Scalbert, C. Morand, C. Remesy and L. Jimenez, 2004. Polyphenols: food sources and bioavailability. Am. J. Clin. Nutr., 79: 727-47.

5. Wang, X., H. He, W. Leng and X. Tang, 2006. Evaluation of brain-targeting for the nasal delivery of estradiol by the microdialysis method, Int. J. Pharm., 317: 40-6.
6. Illum, L., 2000 Transport of drugs from the nasal cavity to the central nervous system. Eur J Pharm Sci., 11: 1-18.

7. Mathison, S., R. Nagilla and U.B. Kompella, 1998. Nasal route for direct delivery of solutes to the central nervous system: factor fiction? J. Drug Target. , 5: 415-41.

8. Cho, J.Y, I.S. Kim, Y.H. Jang, A.R. Kim and S.R. Lee, 2006 Protective effect of quercetin, a natural flavonoid against neuronal damage after transient global cerebral ischemia. Neurosci Lett.,. 404: 330335.

9. Wagner, C., R. Fachinetto, C.L Dalla Corte, V.B. Brito, D. Severo, G. De Oliveira Costa Dias, A.F Morel, C.W. Nogueira and J.B. Rocha, 2006. Quercitrin, a glycoside form of quercetin, prevents lipid peroxidation in vitro, Brain Res., 1107: 192198.

10. de Boer, V.C., A.A. Dihal, H. van der Woude, I.C. Arts, S. Wolffram, G.M. Alink, I.M. Rietjens, J. Keijer and P.C. Hollman, 2005. Tissue distribution of quercetin in rats and pigs. J. Nutr., 135: 1718-25.

11. Manach, C., C. Morand, V. Crespy, C. Demigne, O. Texier, F. Regerat and C. Remesy, 1998. Quercetin is recovered in human plasma as conjugated derivatives which retain antioxidant properties. FEBS Lett., 426: 331-6.

12. Youdim, K.A., M.Z. Oaiser, D.J. Begley, C.A. Rice-Evans and N.J. Abbott, Flavonoid permeability across an in situ model of the bloodbrain barrier, Free Radic Biol Med. , 36: 592-604.

13. Behl, C.R., H.K. Pimplaskar, A.P. Sileno, J. deMeireles and V.D. Romeo., 1998. Effects of physicochemical properties and other factors on systemic nasal drug delivery. Adv. Drug Deliv. Rev., 29: 89-116.

14. Krauze, M.T., J. Forsayeth, J.W. Park and K.S. Bankiewicz, 2006. Real-time imaging and quantification of brain delivery of liposomes. Pharm. Res., 23: 2493-504.

15. Lee, B.H., S.M. Jeong, J.H. Lee, J.H. Kim, I.S. Yoon, J.H. Lee, S.H. Choi, S.M. Lee, C.G. Chang, H.C. Kim, Y. Han, H.D. Paik, Y. Kim and S.Y. Nah, 2005. Quercetin inhibits the 5hydroxytryptamine type 3 receptor-mediated ion current by interacting with pre-transmembrane domain I. Mol. Cells., 20: 69-73. 